ARTIGO ESPECIAL / SPECIAL ARTICLE

\title{
Maior mortalidade durante a pandemia de COVID-19 em áreas socialmente vulneráveis em Belo Horizonte: implicações para a priorização da vacinação
}

\author{
Higher mortality during the COVID-19 pandemic in socially vulnerable \\ areas in Belo Horizonte: implications for vaccine prioritization
}

\author{
Valéria Maria de Azeredo Passos' (D), Luisa Campos Caldeira Brant' (D), Pedro Cisalpino Pinheiro' (D), \\ Paulo Roberto Lopes Correa" (D), Isis Eloah Machado"l' (D), Mayara Rocha Santos" (D), \\ Antonio Luiz Pinho Ribeiro' (D), Lucia Maria Miana Paixão" (D), Fabiano Geraldo Pimenta Junior" (ID, \\ Maria de Fatima Marinho de Souza" (D), Deborah Carvalho Maltav (D)
}

RESUMO: Objetivo: Avaliar a mortalidade por áreas de Belo Horizonte (BH) durante a pandemia de COVID19 conforme a vulnerabilidade social, visando a uma estratégia de vacinação. Métodos: Estudo ecológico com análise de mortalidade, segundo setores censitários classificados pelo índice de vulnerabilidade da saúde, composto de indicadores de saneamento e socioeconômicos. Óbitos por causas naturais e COVID-19 foram obtidos do Sistema de Informação sobre Mortalidade, entre a $10^{\text {a }}$ e a 43 $3^{\text {a }}$ semanas epidemiológicas (SE) de 2020. Calculou-se o excesso de mortalidade por modelo de série temporal, considerando-se as mortes observadas por SE entre 2015 e 2019, por setor censitário. Taxas de mortalidade (TM) foram calculadas e padronizadas por idade com base em estimativas populacionais do Instituto Brasileiro de Geografia e Estatística (IBGE). Resultados: Houve 16,1\% ( $\mathrm{n}=1.524)$ de excesso de mortalidade em BH: 11, 18,8 e 17,3\% nas áreas de baixa, média e elevada vulnerabilidade, respectivamente. As diferenças entre TM observadas e esperadas por causas naturais, padronizadas por idade, foi igual a 59/100 mil habitantes em BH, aumentando de 31 para 77 e $95 / 100$ mil, nas áreas de baixa, média e elevada vulnerabilidade, respectivamente. Houve gradiente de aumento com a idade nas TM por COVID-19, variando de 4 a 611/100 mil habitantes entre as idades de 20-39 anos e 75+ anos. A TM por COVID-19 por 100 mil idosos (60+ anos) foi igual a 292, aumentando de 179 para 354 e 476 nos setores de baixa, média e elevada vulnerabilidade, respectivamente. Conclusão: Desigualdades na mortalidade, mesmo entre idosos, aliadas à baixa oferta de doses, demonstram a importância de priorizar áreas socialmente vulneráveis durante a vacinação contra COVID-19.

Palavras-chave: Mortalidade. Idosos. Infecções por coronavírus. Iniquidade social. Vacinas.

'Faculdade de Medicina, Universidade Federal de Minas Gerais - Belo Horizonte (MG), Brasil.

"Secretaria Municipal de Saúde - Belo Horizonte (MG), Brasil.

"'Escola de Medicina, Universidade Federal de Ouro Preto - Ouro Preto (MG), Brasil.

IV Vital Strategies - São Paulo (SP), Brasil.

vEscola de Enfermagem, Universidade Federal de Minas Gerais - Belo Horizonte (MG), Brasil.

Autor correspondente: Deborah Carvalho Malta. Programa de Pós-Graduação em Enfermagem, Departamento de Enfermagem Materno-Infantil e Saúde Pública, Universidade Federal de Minas Gerais. Avenida Alfredo Balena, 190, Santa Efigênia, CEP: 30130-100, Belo Horizonte, MG, Brasil. E-mail: dcmalta@uol.com.br

Conflito de interesses: nada a declarar - Fonte de financiamento: Vital Strategies. 
ABSTRACT: Objective: To assess mortality during the COVID-19 pandemic according to social vulnerability by areas of Belo Horizonte (BH), aiming at strategies for vaccination. Methods: Ecological study with mortality analysis according to census tracts classified by the Health Vulnerability Index, a composite indicator that includes socioeconomic and sanitation variables. Deaths by natural causes and by COVID-19 were obtained from the "Mortality Information System", between the $10^{\text {th }}$ and $43^{\text {rd }}$ epidemiological weeks (EW) of 2020. Excess mortality was calculated in a time series model, considering observed and expected deaths per EW, between 2015 and 2019 , per census tracts. Mortality rates (MR) were calculated and age-standardized using population estimates from the 2010 census, by the Brazilian Institute of Geography and Statistics (IBGE). Results: Excess mortality in BH was $16.1 \%(\mathrm{n}=1,524): 11,18.8$ and $17.3 \%$ in low, intermediate and high vulnerability areas, respectively. The differences between observed and expected age-standardized MR by natural causes were equal to 59/100,000 inhabitants in $\mathrm{BH}$, increasing from 31 to 77 and 95/100,000 inhabitants in the areas of low, intermediate and high vulnerability, respectively. There was an aging gradient in MR by COVID-19, ranging from 4 to $611 / 100,000$ inhabitants among individuals aged 20-39 years and 75+ years. The COVID-19 MR per 100,000 older adults (60+ years) was 292 in $\mathrm{BH}$, increasing from 179 to 354 and 476, in low, intermediate and high vulnerability areas, respectively. Conclusion: Inequalities in mortality, particularly among older adults, combined with the limited supply of doses, demonstrate the importance of prioritizing socially vulnerable areas during vaccination against COVID-19.

Keywords: Mortality. Aged. Coronavirus infections. Social inequity. Vaccines.

\section{INTRODUÇÃo}

O Brasil é um dos países com maior número de casos e mortes por COVID-19 no mundo, mesmo a mortalidade por COVID-19 sendo subnotificada em, no mínimo, 20\% ${ }^{1}$. Belo Horizonte, a sexta maior capital do país, com 2.423 .737 milhões de habitantes, apresentou 63.387 casos e 1.877 mortes por COVID-19 até dezembro de $2020^{2}$. O município, por meio do monitoramento contínuo da doença, foi pioneiro na instituição de medidas restritivas para contenção da pandemia no Brasil e prontamente iniciou o processo de vacinação ${ }^{3}$.

Determinadas a eficácia e a segurança das diferentes vacinas disponíveis para uso emergencial, a vacinação em massa da população configura-se como a próxima estratégia de enfrentamento da COVID-19, visando à redução da mortalidade e da morbidade pela doença ${ }^{4,5}$. Entretanto, até que a produção de vacinas atinja os níveis exigidos para atender toda a demanda, é preconizada a implementação da vacinação por etapas, com critérios de priorização de grupos instituídos por cada país ${ }^{6}$. O governo brasileiro propôs que a alocação de vacinas contra COVID-19 seja realizada visando à preservação do funcionamento dos serviços de saúde, à proteção dos indivíduos com maior risco de desenvolvimento de formas graves e óbitos, seguidas da preservação do funcionamento dos serviços essenciais e da proteção dos indivíduos com maior risco de infecção ${ }^{7}$.

O Programa Nacional de Imunização (PNI) do Ministério da Saúde tem como princípio a alocação de vacinas para populações-alvo ${ }^{8}$. Entretanto, a distribuição das vacinas geralmente ocorre em situação de disponibilidade de doses. Neste momento de restrição de 
doses da vacina para COVID-19, é preciso discutir os critérios para priorização da vacinação nos grupos considerados prioritários e além deles, a fim de se organizar a logística de implementação da vacinação para a população brasileira, otimizando recursos já existentes.

Estudos no Brasil e no mundo têm demonstrado que a pandemia por COVID-19 atingiu desigualmente as pessoas, com maior número de casos, hospitalizações e mortes entre aquelas com maior vulnerabilidade social ${ }^{9-11}$. Desde 1998 , por reconhecer que o risco de perda de saúde se associa a piores condições ambientais e sociais, a Secretaria Municipal de Saúde de Belo Horizonte (SMS-BH) trabalha com um indicador composto, denominado de índice de vulnerabilidade em saúde (IVS), para a adoção de políticas públicas e a alocação de recursos para os territórios mais vulneráveis da cidade ${ }^{12,13}$.

Com o objetivo de contribuir para a discussão das próximas etapas da vacinação no município de Belo Horizonte, este artigo testou a hipótese de que a população residente em áreas de maior vulnerabilidade social tenha apresentado maior mortalidade na pandemia por COVID-19.

\section{MÉTODOS}

Este é um estudo ecológico, com comparação do perfil da mortalidade por causas naturais e por COVID-19 entre março e outubro de 2020 - da $10^{\mathrm{a}}$ à $43^{\mathrm{a}}$ semana epidemiológica (SE) - por grupo de setores censitários de Belo Horizonte, classificados e agrupados conforme o índice de vulnerabilidade de saúde (IVS).

Todos os óbitos por causas naturais de residentes no município notificados ao Sistema de Informação sobre Mortalidade (SIM) do Ministério da Saúde (MS) foram selecionados e qualificados $^{14}$. Definiu-se temporariamente como causa básica de óbito pela COVID-19 o código B34.2, infecção por coronavírus de localização não especificada, da Décima Revisão da Classificação Internacional de Doenças (CID-10), até a mudança no SIM para a incorporação do novo código para COVID-19 recomendado pela Organização Mundial da Saúde $(\mathrm{OMS})^{15,16}$. Estimativas populacionais por setor censitário foram obtidas do Instituto Brasileiro de Geografia e Estatística (IBGE) ${ }^{17}$.

O IVS em Belo Horizonte, atualizado em 2012 com base nos dados de cada setor censitário do Censo Demográfico de 2010, é composto de cinco indicadores: percentual de saneamento básico, de coleta de lixo e de abastecimento de água, nível de alfabetização e cor da pele dos residentes ${ }^{13}$. Em 2010, Belo Horizonte tinha 2,4 milhões de residentes em 3.830 setores censitários, agrupados de acordo com o IVS em quatro categorias de risco à saúde: baixo (1.330 setores), médio ( 1.460 setores), elevado ( 737 setores) e muito elevado (303 setores $)^{13}$. Como os setores de risco muito elevado representam pequena parcela da população, esta análise foi realizada por grupos de baixa, média e elevada vulnerabilidade, esta última resultante do somatório dos setores de elevada e muito elevada vulnerabilidade.

Para a obtenção do denominador das taxas de mortalidade, estimou-se a população de Belo Horizonte em 2020 para os três grupos de vulnerabilidade em saúde, tomando por base as estimativas do Censo de 2010. Levaram-se em consideração as diferenças de estrutura etária por IVS observadas após a construção das pirâmides etárias com dados do censo 
de 2010 (Figura 1). Primeiramente, calculou-se a taxa de crescimento populacional específica por idade e por sexo para todo o município, com base em projeção populacional já publicada ${ }^{18}$. Essas taxas de crescimento foram então aplicadas aos mesmos estratos de idade e sexo de cada um dos três grupos de IVS (baixo, médio e elevado), obtendo-se, no fim, a estimativa da população em 2020. A diferenciação por sexo foi considerada apenas para a estimativa da população, com as análises de mortalidade realizadas para ambos os sexos.

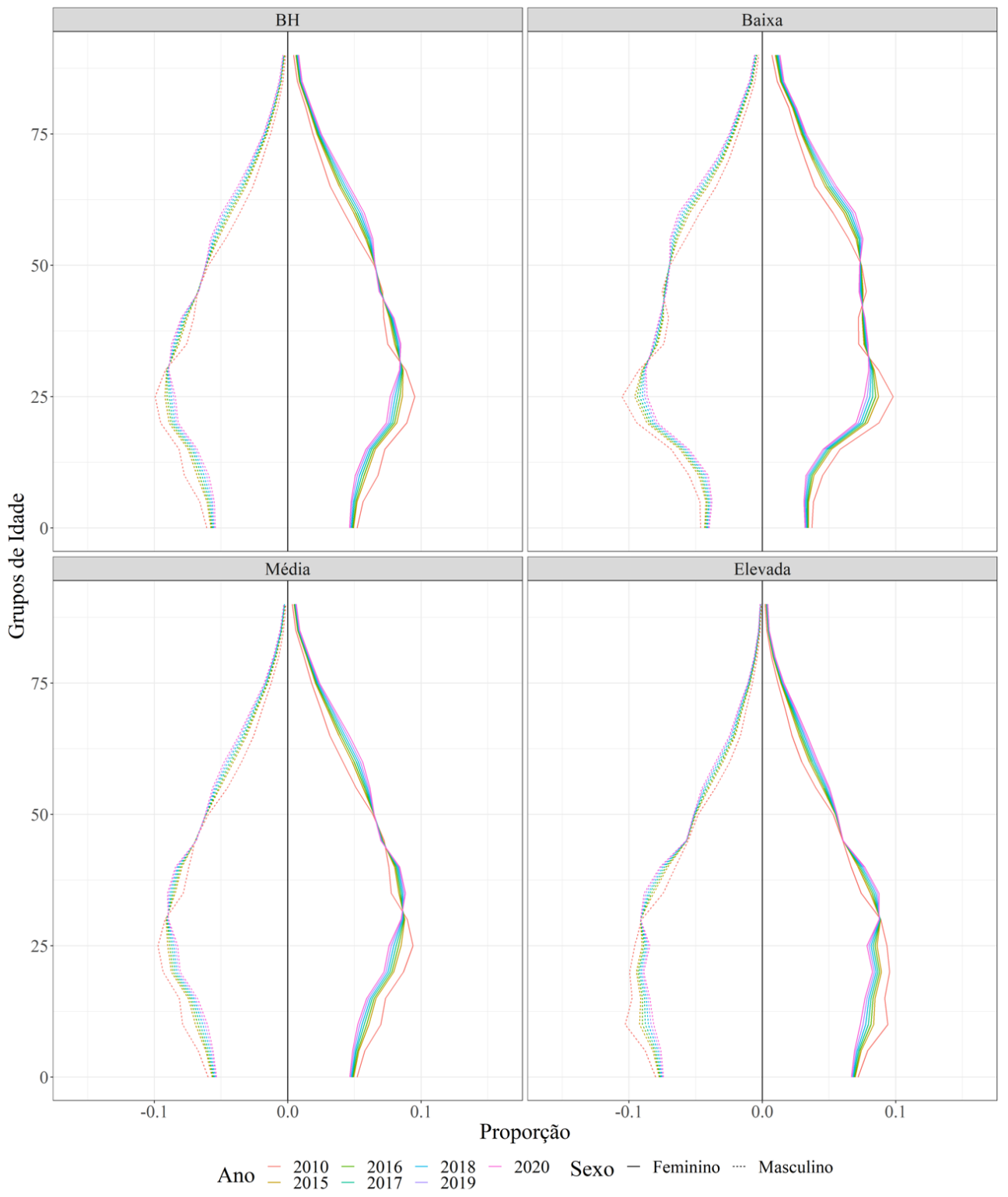

Figura 1. Pirâmides etárias do município de Belo Horizonte e das áreas classificadas pela vulnerabilidade em saúde, de 2010 a 2020. 
O número esperado de mortes por causas naturais para cada categoria de IVS foi estimado aplicando-se o modelo de série temporal autoregressive integrated moving average (ARIMA) ${ }^{19}$ ao número observado de mortes em cada uma das SE de 2015 à $9^{\text {a }}$ SE de 2020, para então projetar o número de mortes entre a $10^{\text {a }}$ e a $43^{\text {a }}$ SE de 2020 . O excesso de óbitos, por SE e por IVS, foi calculado como a diferença entre a média móvel das três últimas semanas do número observado e esperado de óbitos em cada SE. A estimativa das taxas esperadas de mortalidade, por causas naturais e por COVID-19, presumiu que o número projetado (esperado) de óbitos em 2020 , entre a $10^{\mathrm{a}}$ e a $43^{\mathrm{a}} \mathrm{SE}$, apresentasse a mesma distribuição por idade registrada em 2019.

As taxas de mortalidade padronizadas por idade, observadas e esperadas, consideraram os óbitos registrados por IVS para cinco estratos etários: todas as idades; 20-39, 40-59, 60-74 e 75 e mais anos. A padronização direta por idade utilizou como referência a população de Belo Horizonte em 2020. A razão de mortalidade padronizada (mortalidade observada dividida pela mortalidade esperada) e as diferenças entre as taxas de mortalidade (mortalidade observada menos mortalidade esperada) também foram calculadas.

A pesquisa foi apreciada e aprovada pelo Comitê de Ética em Pesquisa da Universidade Federal de Minas Gerais (UFMG) e pela SMS-BH, conforme Certificado de Apresentação para Apreciação Ética (CAAE) nº 39778720.4.3001.5140.

\section{RESULTADOS}

A população de Belo Horizonte foi estimada pelo Censo de 2010 em 2.370.609 habitantes e projetada para 2.423.737 habitantes em 2020, com distribuição percentual semelhante por sexo e por IVS e com média de idade um pouco maior, como esperado para o período. Em 2020, a maior parte da população $(39,8 \%)$ vivia nos setores de média vulnerabilidade, com 34,8 e $25,4 \%$ vivendo nos setores de baixa e elevada vulnerabilidade em saúde, respectivamente. As mulheres foram maioria, 53\% no município, com proporções variando em 52, 53 e 55\% entre os setores de elevada, média e baixa vulnerabilidade. Como esperado, a idade nas áreas de maior vulnerabilidade foi menor. No município, a média de idade em 2020 foi igual a 39 anos (desvio padrão - DP = 21), variando de $43 \pm 22$ anos, $38 \pm 21$ e 33 \pm 21 anos, nos respectivos setores de baixa, média e alta vulnerabilidade.

Em 2020, o número de óbitos observados por causas naturais no município foi $16,1 \%$ superior ao esperado, variando de $11 \%$ nos setores de baixa vulnerabilidade a 18,8 e $17,3 \%$ nos setores de média e elevada vulnerabilidade, respectivamente. A razão padronizada de mortalidade revelou aumento de $16,1 \%$ dos óbitos por causas naturais em Belo Horizonte, com incrementos de 11,1, 18,8 e 19,4\% nos setores de baixa, média e elevada vulnerabilidade. Enquanto as taxas brutas de mortalidade não apresentaram grande variação segundo o IVS e, ao inverso, mostraram-se mais baixas nos setores com IVS elevado (424, 455 e 387 por 100 mil nos setores de IVS baixo, médio e elevado, respectivamente - dados não mostrados), as taxas de mortalidade por causas naturais padronizadas para a idade evidenciaram 
o gradiente de elevação do risco de morte conforme aumenta a vulnerabilidade social (Tabela 1). Isso ocorre para os valores esperados e observados, revelando a maior mortalidade em setores de elevada vulnerabilidade, porém sempre mais altos nos dados observados por causa do impacto da pandemia.

Além disso, esse gradiente foi observado nas diferenças entre as taxas de mortalidade esperada e observada, sugerindo que as disparidades na mortalidade entre os grupos de IVS foram exacerbadas pela pandemia. No período de estudo, para cada 100 mil habitantes, houve o excesso de 59 mortes no município, variando de 31 a 95 entre os setores de baixa e elevada vulnerabilidade (Tabela 1). Ainda nesse período, foram registrados 1.524 óbitos por COVID-19 em Belo Horizonte. A taxa de mortalidade padronizada para a idade por COVID-19 foi igual a 63 por 100 mil habitantes, variando de 35 para 78 e 105 por 100 mil com o aumento da vulnerabilidade em saúde (Tabela 1).

A Figura 2 ilustra a sobreposição entre as médias móveis do excesso de óbitos por causas naturais e dos óbitos por COVID-19 no período analisado, tanto para o município como para a população que vive em setores censitários classificados por cada grupo de IVS. Observa-se o início da pandemia na $10^{\mathrm{a}} \mathrm{SE}$, com pico entre a $25^{\mathrm{a}}$ e a $35^{\mathrm{a}} \mathrm{SE}$. Chama atenção a maior diferença entre as linhas de mortalidade por causas naturais e COVID-19 no pico da pandemia (SE 30) no grupo de elevada vulnerabilidade.

A análise por grupos etários mostra que, no período sob exame, houve aumento consistente da mortalidade observada em relação à esperada, com maiores diferenças entre os óbitos com mais de 60 anos de idade e nos setores de maior vulnerabilidade (Figura 3).

A taxa de mortalidade por COVID-19 por 100 mil idosos $(60+$ anos) foi igual a 292, aumentando de 179 para 354 e 476 nos setores de baixa, média e elevada vulnerabilidade,

Tabela 1. Distribuição da mortalidade por causas naturais e por COVID-19 em Belo Horizonte, por grupos de vulnerabilidade em saúde, entre a 10a e a 43a semana epidemiológica de 2020.

\begin{tabular}{|c|c|c|c|c|}
\hline \multirow{2}{*}{$\begin{array}{l}\text { Indicadores de } \\
\text { Mortalidade }\end{array}$} & \multirow{2}{*}{$\begin{array}{c}\text { Belo } \\
\text { Horizonte }\end{array}$} & \multicolumn{3}{|c|}{ IVS } \\
\hline & & Baixo & Médio & Elevado \\
\hline Óbitos esperados (N) & 8.916 & 3.218 & 3.697 & 2.039 \\
\hline Óbitos observados (N) & 10.356 & 3.573 & 4.391 & 2.392 \\
\hline Excesso de óbitos (\%) & 16,1 & 11 & 18,8 & 17,3 \\
\hline Taxa de mortalidade* esperada & 368 & 281 & 411 & 491 \\
\hline Taxa de mortalidade* observada & 427 & 312 & 489 & 587 \\
\hline $\begin{array}{l}\text { Diferença das taxas de mortalidade } \\
\text { (observada - esperada) }\end{array}$ & 59 & 31 & 77 & 95 \\
\hline $\begin{array}{l}\text { Razão de mortalidade padronizada } \\
\text { (observada/esperada) }\end{array}$ & 116,1 & 111 & 118,8 & 119,3 \\
\hline Óbitos por COVID-19 (N) & 1.524 & 408 & 699 & 417 \\
\hline Taxa de mortalidade* por COVID-19 & 63 & 35 & 78 & 105 \\
\hline
\end{tabular}


respectivamente. A Tabela 2 detalha os aspectos apresentados na Figura 3. Em Belo Horizonte, os valores da razão padronizada de mortalidade revelam pouco ou nenhum impacto da pandemia na mortalidade entre os mais jovens (99,9\%), com aumento de $16,4,23,1$ e $15,5 \%$ em indivíduos com 40-59 anos, 60-74 e 75 e mais anos. Se observados os resultados por grupos de IVS, entre os mais jovens (20-39 anos) a mortalidade observada foi maior que a esperada apenas nos setores de menor vulnerabilidade, com inversão desse padrão com

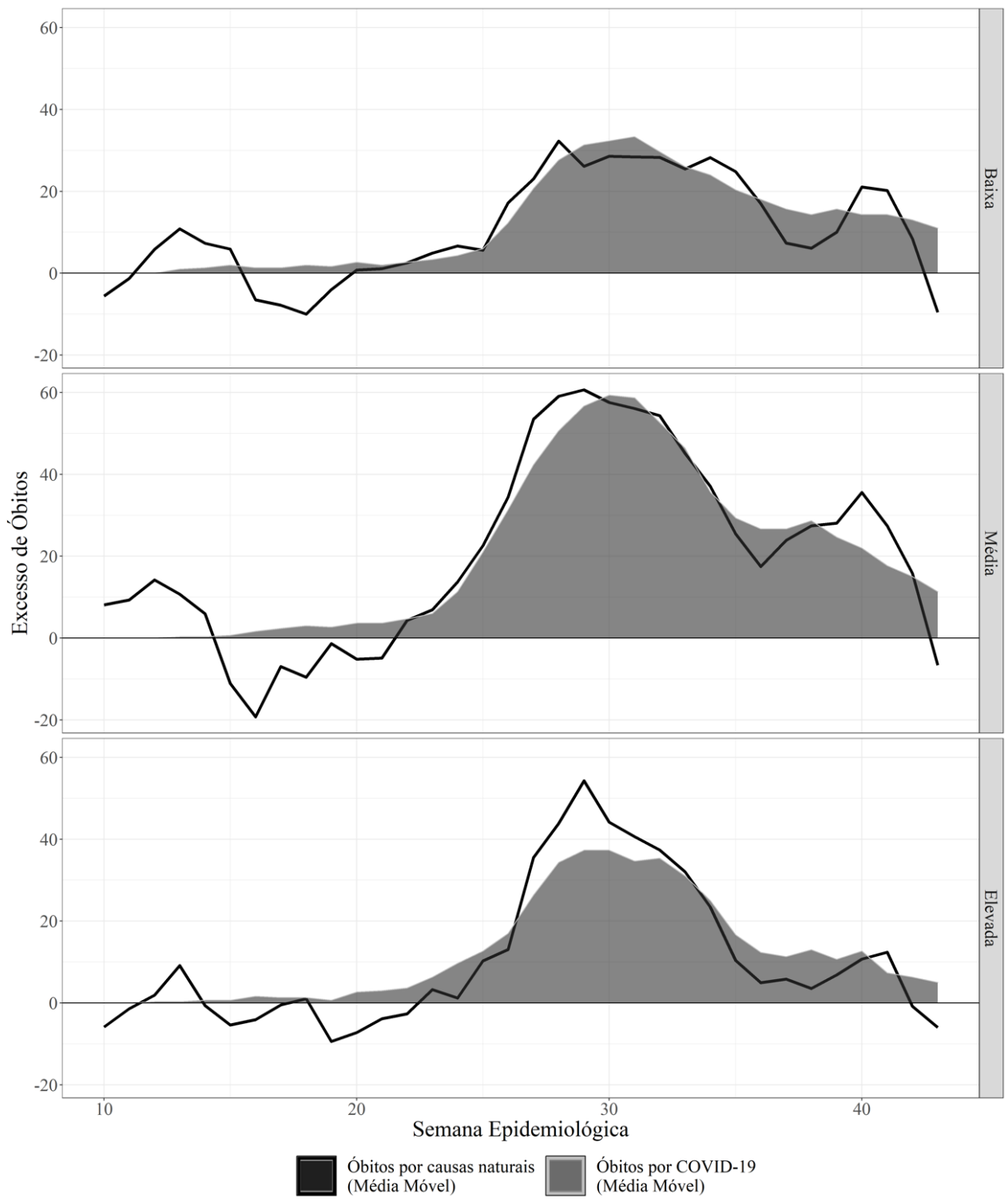

Figura 2. Representação da média móvel do excesso de óbitos por causas naturais e dos óbitos

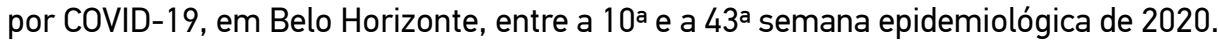


o aumento da idade. Entretanto, quando se observam diferenças entre a razão de mortalidade padronizada do IVS baixo quando comparada às razões do IVS médio e elevado em todas as outras faixas etárias, o impacto da pandemia é evidentemente maior nos grupos de média e alta vulnerabilidade, quando comparados ao de baixa vulnerabilidade. Para idosos de 60-74 anos, por exemplo, a razão de mortalidade padronizada variou 116, 122 e 131,7\% nas áreas com IVS baixo, médio e elevado, respectivamente.

Ficam ainda mais evidentes as disparidades na mortalidade por faixa etária e por IVS quando se analisa a diferença na TM observada em relação à esperada. Ela foi nula apenas para indivíduos de 20-39 anos (-0,02), aumentando para 35, 184 e 562 por 100 mil habitantes entre aqueles com idade entre 40 e 59, 60 e 74 e 75 ou mais anos, respectivamente. Para estes últimos, em geral, as diferenças entre os IVS são mais acentuadas quando os setores com IVS baixo são comparados aos setores com IVS médio ou elevado. Por exemplo, entre adultos de meia

Tabela 2. Distribuição da mortalidade por causas naturais e por COVID-19 entre adultos e idosos de Belo Horizonte, por nível de vulnerabilidade em saúde, entre a $10^{\mathrm{a}}$ e a $43^{\mathrm{a}}$ semana epidemiológica de 2020 .

\begin{tabular}{|c|c|c|c|c|}
\hline \multirow{2}{*}{ Indicador de Mortalidade } & \multirow{2}{*}{$\begin{array}{c}\text { Belo } \\
\text { Horizonte }\end{array}$} & \multicolumn{3}{|c|}{ IVS } \\
\hline & & Baixo & Médio & Elevado \\
\hline \multicolumn{5}{|l|}{ 20-39 anos } \\
\hline TM esperada: TM observada & $36: 36$ & 18: 22 & $37: 36$ & 59: 53 \\
\hline TM observada - TM esperada & $-0,02$ & 4,16 & $-0,7$ & $-5,5$ \\
\hline TM esperada/TM observada* & 99,9 & 123,1 & 98,1 & 90,6 \\
\hline TM por COVID-19 & 4 & 2 & 5 & 6 \\
\hline \multicolumn{5}{|l|}{ 40-59 anos } \\
\hline TM esperada: TM observada & $211: 246$ & 116: 129 & 241: 286 & $337: 382$ \\
\hline TM observada - TM esperada & 35 & 13 & 45 & 45 \\
\hline TM esperada/TM observada & 116,4 & 110,9 & 118,8 & 113,4 \\
\hline TM por COVID-19 & 33 & 12 & 42 & 56 \\
\hline \multicolumn{5}{|l|}{$60-74$ anos } \\
\hline TM esperada: TM observada & 794: 978 & 570: 662 & 912: 1113 & 1101:1450 \\
\hline TM observada - TM esperada & 184 & 91 & 200 & 349 \\
\hline TM esperada/TM observada & 123,1 & 116 & 122 & 131,7 \\
\hline TM por COVID-19 & 164 & 79 & 182 & 331 \\
\hline \multicolumn{5}{|l|}{75 anos ou mais } \\
\hline TM esperada: TM observada & $3.620: 4.182$ & 3148: 3.435 & 4.016: 4.839 & 4.393: 5.242 \\
\hline TM observada - TM esperada & 562 & 287 & 823 & 850 \\
\hline TM esperada/TM observada & 115,5 & 109,1 & 120,5 & 119,3 \\
\hline TM por COVID-19 & 611 & 409 & 800 & 874 \\
\hline
\end{tabular}

TM: taxas de mortalidade padronizadas para a idade, por 100 mil habitantes; *razão padronizada de mortalidade; IVS: índice de vulnerabilidade em saúde. 
idade (40-59 anos), o aumento da TM observada em relação à esperada é igual a 35 por 100 mil em Belo Horizonte, e 13, 45 e 45 por 100 mil nos respectivos grupos de baixa, média e elevada vulnerabilidade. Entre idosos, o gradiente de aumento entre os IVS é mais claro: entre aqueles com 60-74 anos, a diferença das TM observadas e esperadas é igual a 184 por 100 mil em Belo Horizonte, variando de 91 a 200 e 349 por 100 mil entre os três grupos de vulnerabilidade. Em relação às TM por COVID-19, houve claro gradiente de aumento com a idade, que variou de 4, 33, 164 a 611 por 100 mil entre as respectivas faixas etárias de 20-39, 40-59,

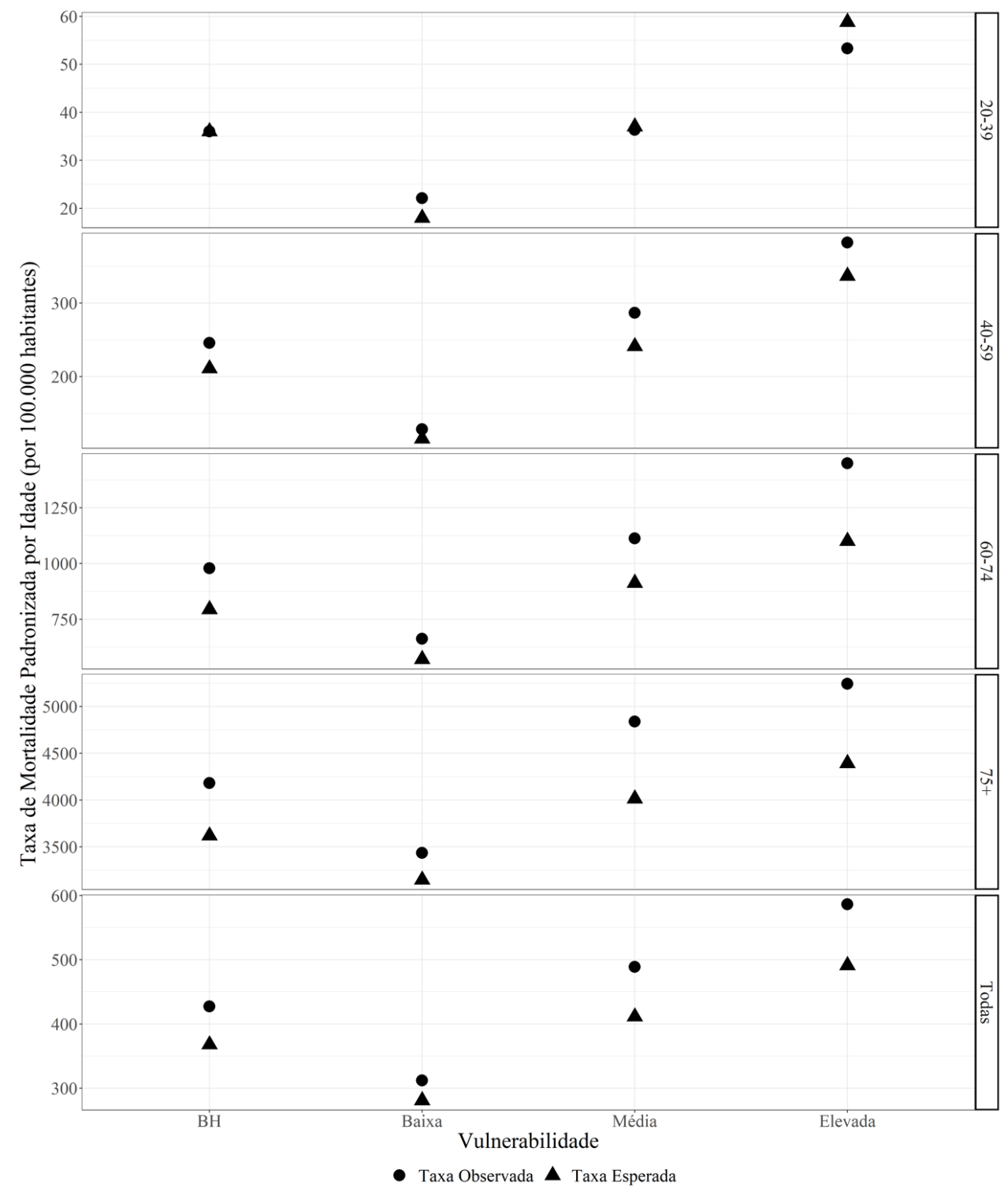

Figura 3. Taxas de mortalidade padronizadas por 100 mil habitantes por causas naturais, por faixas etárias e por todas as idades, em Belo Horizonte, entre a 10a e a 43a semana epidemiológica de 2020. 
60-74 e 75 e mais anos. O diferencial de mortalidade entre os IVS foi maior com o aumento da idade, e as TM entre os mais idosos (75+ anos) foram de 408, 800 e 874 por 100 mil, respectivamente, nos três grupos de vulnerabilidade, centenas de vezes maiores que as taxas entre os mais jovens (20-39 anos), iguais a 2, 5 e 6 por 100 mil para os mesmos grupos.

\section{DISCUSSÃO}

Este estudo confirma a hipótese de aumento diferencial da mortalidade por causas naturais e por COVID-19 em populações com maior vulnerabilidade social em Belo Horizonte em 2020 e, principalmente, entre idosos, com implicações urgentes relativas à estratégia de vacinação da população do município.

No período de estudo, o excesso de mortalidade por causas naturais tem como principal responsável a pandemia por COVID-19, como mostra a sobreposição entre as médias móveis da mortalidade por causas naturais e por COVID-19 no município. Em um contexto de baixa testagem para identificação de casos, assim como de falta de padronização da certificação de óbitos, o excesso de mortalidade é a ferramenta indicada para garantir aos gestores de saúde um indicador confiável da magnitude da pandemia por refletir os seus impactos diretos e indiretos na mortalidade de uma localidade ${ }^{20}$.

O excesso de mortes por causas naturais em Belo Horizonte foi de 16,1\%, maior nos setores de média e elevada vulnerabilidade. Porém, identificamos que o número de mortes por COVID-19 foi responsável por uma parcela menor do excesso de mortes por causas naturais nas áreas de elevada vulnerabilidade, em relação às áreas de baixa e média vulnerabilidade. Isso sugere um possível subdiagnóstico de COVID-19 nessas áreas, potencialmente em razão da desigualdade de acesso aos serviços de saúde e aos testes diagnósticos, como já revelado em outras cidades brasileiras ${ }^{21}$. Alternativamente, pode ter ocorrido um aumento adicional de mortes naturais por outras causas naturais como consequência indireta da pandemia, nas áreas de maior vulnerabilidade social ${ }^{22}$.

A razão de mortalidade padronizada, relação entre mortalidade esperada e observada, responde a importante questão etiológica ao demonstrar que $16 \%$ do excesso de óbitos em Belo Horizonte foi causado, direta ou indiretamente, pela pandemia. Entretanto, não responde à pergunta importante para os gestores relativa ao impacto da introdução de medidas para redução da mortalidade, entre elas a vacinação da população. Esse impacto foi evidenciado no presente estudo pela diferença entre a TM observada e esperada por COVID-19, três vezes maior (95 por 100 mil habitantes) nos setores de elevada vulnerabilidade quando comparada aos de menor vulnerabilidade (31 por 100 mil habitantes).

Desigualdades socioeconômicas são os principais determinantes da ocorrência e distribuição das doenças e mortes no mundo e, em Belo Horizonte, essa desigualdade - que já era nítida antes da pandemia — foi claramente exacerbada por ela ${ }^{23,24}$. Pessoas que moram em áreas socialmente mais vulneráveis não só constituem o grupo populacional com maior risco de exposição por geralmente trabalharem em serviços essenciais, mas também por terem dificuldades de distanciamento físico e higiene inerentes às suas condições de moradia e transporte. Além disso, 
as comorbidades que aumentam a letalidade da COVID-19, tais como doenças cardiovasculares, diabetes e obesidade, são mais prevalentes em grupos com piores condições socioeconômicas ${ }^{25}$. Por último, há ainda o menor acesso da população mais vulnerável aos serviços de saúde — disparidade também acentuada pela pandemia. Assim, os dados deste estudo reforçam a necessidade de incluir critérios socioeconômicos na definição da priorização das vacinas.

Em relação aos grupos etários, as desigualdades na mortalidade estão presentes, mesmo que em graus diferentes, nas pessoas com mais de 40 anos, nas quais a letalidade pela COVID19 já é sabidamente maior que nas mais jovens ${ }^{26}$. Assim, os achados da presente pesquisa são consistentes com a maior mortalidade entre idosos encontrada em outros países. Nos EUA, a letalidade por COVID-19 variou de 10 a $27 \%$ para idosos com 85 ou mais anos e de 3 a $11 \%$ para idosos de 65-84 anos, mas teve taxas inferiores a 1\% entre adultos com idades entre 20 e 54 anos $^{26}$. Porém, nosso estudo acrescenta que entre idosos mais vulneráveis as taxas de mortalidade foram muitas vezes mais elevadas que nos outros grupos, o que reforça a necessidade da priorização da vacina para esse grupo em quadro de escassez de doses. Mesmo em países desenvolvidos, já se discute que desconsiderar a heterogeneidade socioeconômica entre idosos pode contribuir para aprofundar as desigualdades sociais ${ }^{24}$.

A metodologia deste estudo é um ponto forte a ser ressaltado. As diferenças entre as estruturas etárias por IVS foi considerada quando se realizou a projeção demográfica. Além disso, a padronização das taxas de mortalidade por idade permitiu a comparação entre os grupos de IVS com diferentes estruturas etárias, levando à comprovação de maior mortalidade entre os idosos dos setores mais vulneráveis, que geralmente concentram menor número de idosos e, consequentemente, de óbitos ${ }^{27}$.

Esta investigação apresenta limitações inerentes ao seu desenho. Há provável subestimação dos óbitos por COVID-19 obtidos do SIM, que pode implicar também a subestimativa da real diferença entre a mortalidade por COVID-19 nas áreas socialmente vulneráveis, com menor acesso ao diagnóstico e atenção em saúde. ${ }^{1} \mathrm{~A}$ análise foi realizada apenas até a $43^{\mathrm{a}} \mathrm{SE}$, última SE com dados atualizados, revisados e disponibilizados pela SMS-BH, mas é improvável que o acréscimo de outras mude a direção ou a magnitude dos achados. A taxa de crescimento aplicada para determinação da população de cada setor censitário foi uniforme, baseada no único estudo demográfico disponível para o município ${ }^{18}$. Apesar de se esperar ritmo de crescimento populacional desigual ente as áreas de IVS, na ausência de informações atualizadas, entendemos que a aproximação adotada oferece o melhor parâmetro possível para a distribuição etária nos estratos populacionais de interesse. Apesar de os intervalos de confiança das estimativas não terem sido calculados, tanto a magnitude como os gradientes das diferenças entre os grupos comparados sugerem fortemente que as discrepâncias encontradas sejam reais. Também salientamos que o IVS é calculado para um conjunto de pessoas residentes em uma determinada área geográfica contínua, não se identificando diferenças entre seus moradores. Os parâmetros utilizados para a composição desse índice referem-se ao último censo de 2010 . Mesmo que tenha havido mudanças no período, este estudo revela que a desigualdade persiste.

Em suma, o presente trabalho aponta para a maior mortalidade durante a pandemia de COVID-19 em áreas de vulnerabilidade social elevada e média, sobretudo entre os idosos. Assim, recomenda-se que as próximas etapas de vacinação priorizem áreas de maior vulnerabilidade 
social em Belo Horizonte — onde ocorreram quase dois terços das mortes da cidade —, mantidas as demais prioridades propostas pelo governo brasileiro, como estratégia para um impacto maior e mais precoce na morbimortalidade pela doença no município. Salientamos a importância dessa proposta tanto pelo melhor aproveitamento das doses disponíveis da vacina como pela sua exequibilidade, dada a experiência do município em articular ações de vigilância em saúde com bases territoriais definidas em áreas de abrangência do Programa de Saúde da Família ${ }^{28}$.

A administração de Belo Horizonte foi pioneira na adoção de medidas restritivas visando proteger a população. Priorizar a vacinação por idosos das áreas mais vulneráveis trará ganhos para todos os cidadãos do município, pois além da maior redução de mortes haverá maior racionalidade de gastos e de disponibilidade de recursos em saúde.

\section{REFERÊNCIAS}

1. Marinho MF. Semelhanças e diferenças na interpretação dos dados sobre SG, SRAG e COVID-19: SIM, SIVEPGripe e Cartórios de Registro Civil. In: Santos AO, Lopes TL, editores. Coletânea CONASS, Planejamento e Gestão. Brasília: Conselho Nacional de Secretários de Saúde; 2021. v. 2. p. 112-25.

2. Secretaria Municipal de Saúde de Belo Horizonte. Boletim Epidemiológico e Assistencial COVID-19, 176/2020 [Internet]. Belo Horizonte: Secretaria Municipal de Saúde de Belo Horizonte; 2020 [acessado em 8 fev. 2021] Disponível em: https:// prefeitura.pbh.gov.br/sites/default/files/estruturade-governo/saude/2020/boletim_epidemiologico_ assistencial_176_covid-19_31-12-2020.pdf

3. Tupinambás U, Alvim, CG, Oliveira B. A falsa polêmica entre a "bolsa" e a vida sobre isolamento social em Belo Horizonte [Internet]. 2020 [acessado em 28 jan. 2021]. Disponível em: https://ufmg.br/ storage/0/b/5/1/0b5157701a2ebb4c62145cb6cb0 13d20_15880402200862_1907396331.pdf

4. Schaffer DeRoo S, Pudalov NJ, Fu LY. Planning for a COVID-19 Vaccination Program. JAMA. 2020;323(24):2458-9. http:// doi.org/10.1001/ jama.2020.8711

5. World Health Organization. Status of COVID-19 Vaccines within WHO EUL/PQ evaluation process [Internet]. 2021 [acessado em 8 fev. 2021]. Disponível em: https: / / extranet.who.int/pqweb/sites/default/files/ documents/Status_COVID_VAX_20Jan2021_v2.pdf

6. Organização Mundial da Saúde. Concepção da OMS sobre acesso justo e distribuição equitativa de produtos de saúde contra a COVID-19 [Internet]. Organização Mundial da Saúde; 2021 [acessado em 8 fev 2021]. Disponível em: https: / www.who.int/ docs/ defaultsource / coronaviruse / 202485-covid-19-e-2-pt.pdf
7. Brasil. Ministério da Saúde. Plano nacional de operacionalização da vacinação contra a COVID-19 [Internet]. Brasília: Ministério da Saúde; 2021 [acessado em 30 jan. 2021]. Disponível em: https: / / www.gov. $\mathrm{br} / \mathrm{saude} / \mathrm{pt}-\mathrm{br} / \mathrm{media} / \mathrm{pdf} / 2020 /$ dezembro/16/ plano_vacinacao_versao_eletronica.pdf

8. Brasil. Ministério da Saúde. Programa Nacional de Imunização: 30 anos. Brasília: Ministério da Saúde; 2003 [acessado em 30 jan. 2021]. 206 p. Disponível em: http: / /bvsms.saude.gov.br/bvs/publicacoes / livro_30_anos_pni.pdf

9. Hallal PC, Hartwig FP, Horta BL, Silveira MF, Struchiner CJ, Vidaletti LP, et al. SARS-CoV-2 antibody prevalence in Brazil: results from two successive nationwide serological household surveys. The Lancet 2020; 8(11): E1390-8. https:/ / doi.org/10.1016/ S2214-109X(20)30387-9

10. De Negri F, Galiezz R, Miranda P, Koeller P, Zucoloto G, Costa J, et al. Socioeconomic factors and the probability of death by Covid-19 in Brazil. J Public Health 2021; fdaa279. https: / / doi.org/10.1093/pubmed/fdaa279

11. Butler SM. Four COVID-19 Lessons for Achieving Health Equity. JAMA Health Forum 2020; 324(22): 2245-6. https: / / doi.org/10.1001/ jama.2020.23553

12. Boccolini CS, Souza Junior PRB. Inequities in Healthcare utilization: results of the Brazilian National Health Survey, 2013. Int J Equity Health 2016; 15: 150. https: / / doi.org/10.1186/s12939-016-0444-3

13. Secretaria Municipal de Saúde de Belo Horizonte. Gerência de Epidemiologia e Informação. Índice de Vulnerabilidade da Saúde 2012. Belo Horizonte: Secretaria Municipal de Saúde de Belo Horizonte; 2013 [acessado em 30 jul. 2020]. Disponível em:https:/ / prefeitura.pbh.gov.br/sites/default/ files/estrutura-de-governo/saude/2018/publicacaoes-davigilancia-em-saude/indice_vulnerabilidade2012.pdf 
14. Brasil. Ministério da Saúde. Sistema de Informação sobre Mortalidade [Internet]. Brasil: Ministério da Saúde; 2008 [acessado em 8 fev. 2021]. Disponível em: http:/ / www2. datasus.gov.br/DATASUS $/$ index.php?area $=060701$

15. Brasil. Ministério da Saúde. Orientações para codificação das causas de morte no contexto da COVID-19. Brasil: Secretaria de Vigilância em Saúde. Departamento de Análise em Saúde e Vigilância de Doenças não Transmissíveis; 2020 [acessado em 30 jul. 2020]. Disponível em: https: / / portalarquivos2.saude.gov.br/images/pdf/2020/April/29/ Nota-Informativa-declara----o-obito.pdf

16. Organização Mundial da Saúde. Diretrizes internacionais para a certificação e classificação (codificação) da covid19 como causa de morte [Internet]. Organização Mundial da Saúde; 2020 [acessado em 8 fev. 2021]. Disponível em: https: / / www.who.int/ classifications / icd/Guidelines_Cause_of_Death_COVID-1920200420-PT_Apr_24.pdf

17. Brasil. Ministério da Economia. Instituto Brasileiro de Geografia e Estatística. Base de dados do Censo Demográfico 2010: Resultados do universo por setor censitário [Internet]. Brasil: Instituto Brasileiro de Geografia e Estatística; 2011 [acessado em jun. 2020]. Disponível em: ftp: / ftp.ibge.gov.br/Censos/ Censo_Demografico_2010/Resultados_do_Universo/ Agregados_por_Setores_Censitarios /

18. Freire FHMA, Gonzaga MR, Queiroz BL. Projeção populacional municipal com estimadores bayesianos, Brasil 2010 - 2030. In: Sawyer DO, editor. Seguridade Social Municipais. Projeto Brasil 3 Tempos. Brasília: Secretaria Especial de Assuntos Estratégicos da Presidência da República (SAE/SG/PR), United Nations Development Programme (UNDP), International Policy Centre for Inclusive Growth; 2019.

19. Hyndman RJ, Athanasopoulos G. Forecasting: principles and practice. $2^{\mathrm{a}}$ ed. [Internet]. Melbourne: OTexts; 2018 [acessado em 8 fev. 2021]. Disponível em: https: / / otexts.com/fpp2/

20. Vital Strategies, World Health Organization. Revealing the Toll of COVID-19: A Technical Package for Rapid Mortality Surveillance and Epidemic Response. Nova York: Vital Strategies; 2020.

21. Sousa TCM, Moreira NP, KriegerJE, Rosa ISC, Zamudio MM, Veras MASM, et al. Using RT-PCR Testing to Assess the Effectiveness of Outbreak Control Efforts in São Paulo State, the Pandemic's Epicenter in Brazil, according to Socioeconomic Vulnerabilities. medRxiv [Internet]. 2020 [acessado em 8 fev. 2021]. https: / / doi. org/10.1101/2020.10.29.20221960

22. Brant LCC, Nascimento BR, Teixeira RA, Lopes MACQ, Malta DC, Oliveira GMM, et al. Excess of cardiovascular deaths during the COVID-19 pandemic in Brazilian capital cities. Heart 2020; 106: 1898-905. https: / / doi.org/10.1136/heartjnl-2020-317663

23. Corrêa PRL, Ishitani LH, Abreu DMX, Teixeira RA, Marinho F, França EB. The importance of surveillance in cases and mortality from COVID-19 epidemic in Belo Horizonte, 2020. Rev Bras Epidemiol 2020; 23. https: / / doi.org/10.1590/1980-549720200061

24. Jean-Jacques M, Bauchner H. Vaccine DistributionEquity Left Behind? JAMA 2021; 325(9): 829-30. https: / / doi.org/10.1001/jama.2021.1205

25. Williamson EJ, Walker AJ, Bhaskaran K, Bacon S, Bates C, Morton CE, et al. Factors associated with COVID19-related death using Open SAFELY. Nature 2020; 584 : 430-6. https: / / doi.org/10.1038/s41586-020-2521-4

26. CDC COVID-19 Response Team. Severe Outcomes Among Patients with Coronavirus Disease 2019 (COVID-19) - United States, February 12-March 16, 2020. MMWR Morb Mortal Wkly Rep 2020; 69(12): 343-6. https: / / doi.org/10.15585/ mmwr.mm6912e2

27. Queiroz BL, Freire FHMA, Lima EEC, Gonzaga MR. O papel da estrutura etária na análise da mortalidade por Covid-19. Ciênc. Saúde Coletiva 2020; 25(9): 3689-90. http:/ / dx.doi.org/10.1590/1413-81232020259.19682020

28. Belo Horizonte. Secretaria Municipal de Saúde. Carteira Orientadora de Serviços do SUS-BH [Internet]. Belo Horizonte: Secretaria Municipal de Saúde; 2018 [acessado em 8 fev. 2021]. Disponível em: https:// prefeitura.pbh.gov.br/sites/default/files/estrutura-degoverno/saude/carteira-orientadora_servi\%C3\%A7ossus-bh-07-11-2018.pdf

Recebido em: 22/02/2021

Revisado em: 16/03/2021

Aceito em: 01/04/2021

Preprint em: 05/04/2021

Contribuição dos autores: Valéria M A Passos liderou a conceituação, a metodologia, a supervisão das análises, a redação e a edição do manuscrito. Luisa C C Brant, Paulo R L Corrêa, Isis E Machado, Mayara R Santos, Deborah C Malta colaboraram na conceituação, na metodologia, na supervisão das análises, na edição e na revisão do manuscrito. Pedro C Pinheiro colaborou na metodologia, na edição e na revisão do manuscrito, além de realizar as análises estatísticas. Antonio L P Ribeiro, Lucia M M Paixão, Fabiano G P Júnior e Maria de Fatima M de Souza colaboraram na redação, na edição e na revisão do manuscrito. Todos os autores aprovaram a versão final do artigo. 